\title{
REVIEW
}

\section{Non-infectious pulmonary complications after bone marrow transplantation}

\author{
I Khurshid, L C Anderson
}

Postgrad Med J 2002;78:257-262

Bone marrow transplantation (BMT) is a successful and recognised treatment option for patients with a number of haematological and non-haematological malignant and non-malignant conditions. Pulmonary complications both infectious and non-infectious are common after BMT. Multiple factors are thought to contribute to pulmonary complications, including the type and duration of immunological defects produced by the underlying disease and treatment, the development of graft-versus-host disease (GVHD), and the conditioning regimens employed. These complications are classified as early or late, depending on whether they occur before or after 100 days from transplantation. Early non-infectious pulmonary complications typically include pulmonary oedema, upper airway complications, diffuse alveolar haemorrhage, cytolytic thrombi, and pleural effusion. Bronchiolitis obliterans, veno-occlusive disease, and secondary malignancies occur late after BMT. Idiopathic pneumonia syndrome, GVHD, and radiation induced lung injury can occur in early or late period after BMT.

See end of article for authors' affiliations

...............

Correspondence and reprint requests to: $\mathrm{Dr}$ Imtiaz Khurshid,

Department of Pulmonary and Critical Care

Medicine, Brody School of Medicine at East Carolina University, Greenville, NC 27858, USA;

khurshidi@mail.ecu.edu

Submitted 22 June 2001 Accepted

7 January 2001
S ince the first successful allogeneic BMT in the late 1960s, BMT has been used with increasing frequency to treat malignant and non-malignant haematological disorders, solid tumours, metabolic, and genetic diseases. Pulmonary complications, including infectious and non-infectious, occur in $40 \%-60 \%$ of all BMT recipients, which accounts for a considerable morbidity and mortality. ${ }^{1}$ The incidence of noninfectious pulmonary complications has been reported as high as $65 \%$ in allogeneic BMT recipients. ${ }^{2}$ Multiple factors thought to contribute to pulmonary complications, including immunological defects secondary to underlying disease and its treatment, conditioning regimen, and development of graft-versus-host disease (GVHD).. Pulmonary complications are classified into early (box 1) or late (box 2) depending on their onset, whether they occur before or after 100 days after transplantation (fig 1).

\section{UPPER AIRWAY COMPLICATIONS}

These are the earliest complications and usually result from mucosal damage by conditioning regimen. High dose chemotherapy and total body irradiation can cause severe mucosal damage/ mucositis and can lead to upper airway inflammation, laryngeal oedema, dysphagia, odynophagia, or aspiration pneumonitis. ${ }^{4}$ Management of upper airway complications may require from local symptomatic care to intubation and mechanical ventilation for laryngeal oedema in severe cases.

\section{PULMONARY OEDEMA}

The onset of pulmonary oedema after BMT is rapid and is usually seen in the second or third week after BMT. ${ }^{5}$ The exact incidence is not known but it is not an uncommon problem. ${ }^{5}$ It presents as acute onset dyspnoea, weight gain, bibasilar crackles, and evidence of hypoxaemia on arterial blood gas. The chest radiograph shows mild to severe vascular redistribution, diffusely increased vascular marking, and occasionally the presence of pleural effusion. Chest radiographic findings are indistinguishable from pulmonary oedema of any other aetiology (fig 2).

Increased capillary hydrostatic pressure, increased capillary permeability, or a combination of both result in pulmonary oedema. Aggressive hydration to minimise the toxicity of chemotherapeutic agents, blood and blood product transfusion, total parenteral nutrition, cardiac and renal toxicity from chemotherapeutic agents (that is, doxorubicin, cyclophosphamide, cisplatin, cyclosporin) can contribute to the development of cardiogenic pulmonary oedema secondary to increased capillary hydrostatic pressure. Total body irradiation, lung injury from immunosuppressive medications and sepsis can result in increased capillary permeability and lead to noncardiogenic pulmonary oedema. ${ }^{6}$

The management of post-BMT pulmonary oedema is similar to the management of pulmonary oedema of any other aetiology. The development of pulmonary oedema can be prevented by careful physical examination, monitoring weight change, judicious fluid administration, and careful administration of diuretics. ${ }^{6}$ Some patients can develop respiratory failure and need mechanical ventilation.

\section{IDIOPATHIC PNEUMONIA SYNDROME}

Idiopathic pneumonia syndrome (IPS) is defined as diffuse lung injury occurring after BMT for which an infectious or non-infectious aetiology cannot be found. ${ }^{7}$ Previously used histological

Abbreviations: BMT, bone marrow transplantation; $D A H$, diffuse alveolar haemorrhage; GVHD, graft-versus-host disease; IPS, idiopathic pneumonia syndrome 


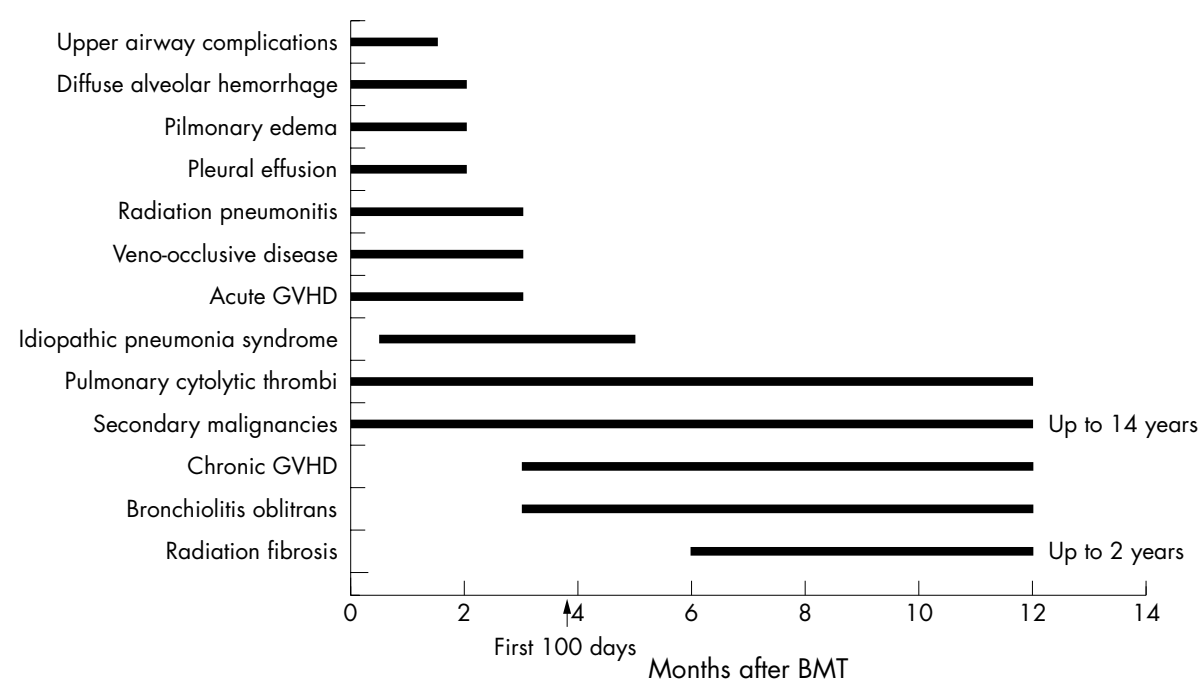

Figure 1 The chronology of non-infectious pulmonary complications after BMT.

\section{Box 1: Early complications (within 100 days of BMT)}

- Pulmonary oedema

- Upper airway complications.

- Idiopathic pneumonia syndrome.

- Diffuse alveolar haemorrhage.

- Radiation pneumonitis.

- Acute GVHD.

- Pleural effusion

- Cytolytic thrombi.

- Mediastinal emphysema.

\section{Box 2: Late complications (after 100 days of BMT)}

- Chronic GVHD

- Bronchiolitis obliterans.

- Idiopathic pneumonia syndrome.

- Secondary alveolar proteinosis.

- Secondary malignancies.

- Radiation fibrosis.

- Veno-occlusive disease.

terms such as interstitial pneumonia, a histological term in reference to this entity, is inaccurate and should be avoided. The two main histopathological patterns of IPS are interstitial pneumonitis and diffuse alveolar damage. ${ }^{7}$ The incidence of IPS after allogeneic BMT is approximately $12 \%$. The classic presentation includes dyspnoea, non-productive cough, hypoxaemia, and non-lobar radiographic infiltrates (fig 3) but clinical presentation can vary from asymptomatic to full blown acute respiratory distress syndrome. The median time of onset is $42-49$ days after BMT, but there is an early peak in the first 14 days after BMT, followed by a lower but consistent incidence up to 80 days. $^{7}$

The diagnosis of IPS is usually one of exclusion; however in 1993 National Heart Lung and Blood Institute workshop proposed the following criteria for the diagnosis of IPS: symptoms and signs of pneumonia, evidence of abnormal pulmonary physiology such as increased alveolar to arterial oxygen gradient or increased restrictive pulmonary test physiology, evidence of widespread alveolar injury suggested by multilobar infiltrates on chest radiography or computed tomography, and absence of active lower, respiratory tract infection. ${ }^{7}$ Appropriate evaluation includes bronchoalveolar lavage or transbronchial biopsy showing no bacterial, viral, or fungal pathogens on culture and cytology and a negative second con-

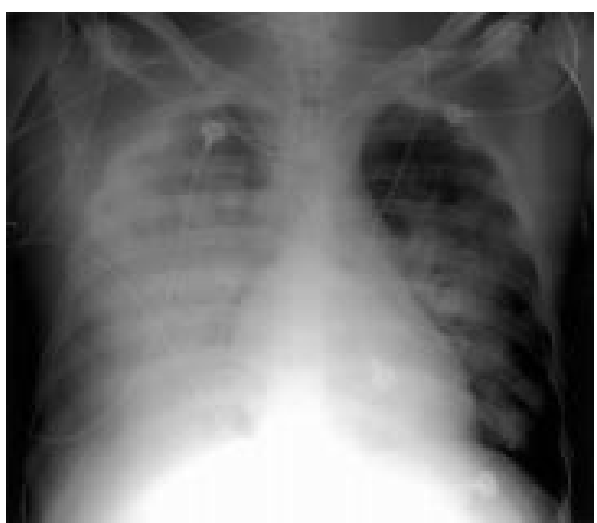

Figure 2 Chest radiograph of a 24 year old woman who underwent autologous BMT for Hodgkin's disease, showing pulmonary oedema and right sided pleural effusion.

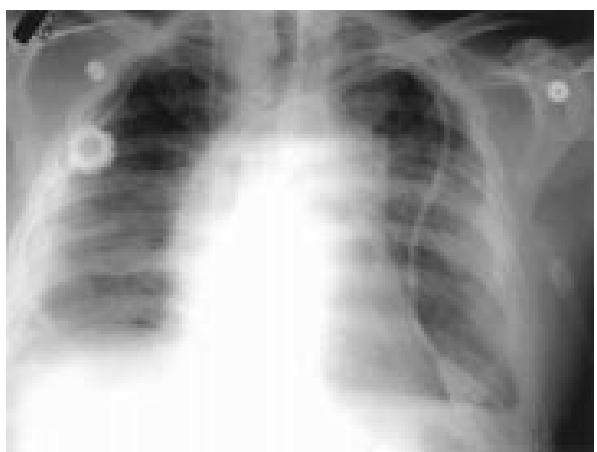

Figure 3 Chest radiograph of a 51 year old man who underwent autologous BMT for non-Hodgkin's lymphoma, showing bilateral interstitial infiltrates. Bronchoalveolar lavage and open lung biopsy did not disclose an infectious or non-infectious cause for pulmonary infiltrates.

firmatory test for infection performed two to 14 days after the initial negative test.

There is no specific treatment for IPS and corticosteroids have no proved efficacy in treatment or prophylaxis. ${ }^{8}$ There is reduced severity of disease in patients receiving cyclosporin or intravenous immunoglobulin prophylaxis for GVHD. ${ }^{9}$ The majority of patients with IPS die from recurrent respiratory failure after initial improvement or from superimposed bacterial, viral, or fungal infections. ${ }^{5}$ Surprisingly patients who 


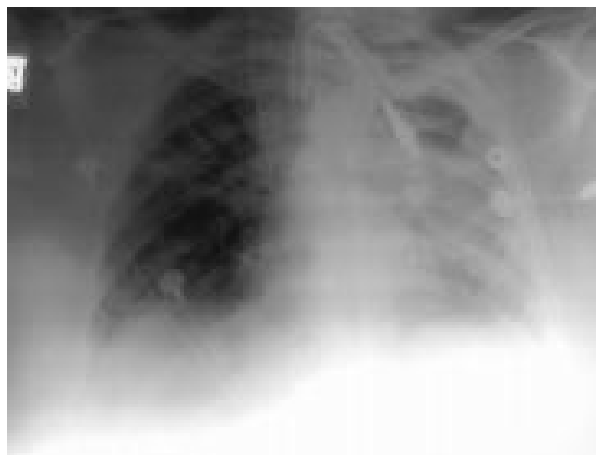

Figure 4 Chest radiograph of a 21 year old woman, who had diffuse alveolar haemorrhage after autologous BMT, showing diffuse left sided alveolar infiltrates.

received total fractioned irradiation in excess of $1200 \mathrm{rad}$ and those with the evidence of acute GVHD (>grade 2) have a better prognosis. ${ }^{8}$

\section{DIFFUSE ALVEOLAR HAEMORRHAGE}

Diffuse alveolar haemorrhage (DAH) is a serious post-BMT complication that has been reported predominately in autologous BMT recipients but it can also occur after allogeneic BMT. $^{10} 11$ The reported incidence of DAH in autologous BMT recipients is $7 \%-14 \% .{ }^{12}$ The syndrome usually occurs in the second and third weeks (range between 5-34 day) after the transplantation. ${ }^{10}$ The exact cause of DAH is unknown. Age $>40$, radiation toxicity, solid malignancy, drug toxicity, infection, thrombocytopenia, and neutrophil influx into the lungs all have been implicated in the pathogenesis of DAH. ${ }^{10}{ }^{12}$ Another study finds no relationship between these variables and the presence of $\mathrm{DAH} .{ }^{13}$ A recent study finds an association between microangiopathy and DAH after BMT. ${ }^{14}$

The characteristic clinical features include sudden onset of progressive dyspnoea, non-productive cough, fever, and hypoxaemia. Haemoptysis is a rare finding. ${ }^{12}$ The radiographic findings are non-specific and cannot be distinguished from pulmonary haemorrhage of any other aetiology. ${ }^{12}$ Most patients initially show a mild interstitial or alveolar infiltrates in the central and lower lung zones, which are indistinguishable from pulmonary oedema or opportunistic infection (fig 4). Characteristically DAH is a fulminant process and radiographic changes rapidly progress to a diffuse, severe alveolar pattern. ${ }^{12}$

The diagnosis is usually made on bronchoscopy, on the basis of the result of bronchoalveolar lavage, which shows a progressive diffusely haemorrhagic fluid. Although the most appropriate diagnostic approach is open lung biopsy, the critical condition of these patients with suspected DAH almost always exclude this option. ${ }^{14}$ One of the diagnostic criteria for DAH on lung biopsy is the presence of pure blood in at least $30 \%$ of the evaluated alveolar surface area in the absence of any concomitant pathology.

The early diagnosis of DAH and prompt intervention is crucial and it may favourably alter the natural history of the disease. $^{101115}$ Administration of high dose corticosteroids improves the total survival, survival to hospital discharge, and reduces the development of subsequent respiratory failure. ${ }^{14}{ }^{15}$ Despite treatment, DAH is associated with very high in hospital mortality $(67 \%$ treated with high dose steroids $v 90 \%$ supportive care).$^{15}$

\section{RADIATION PNEUMONITIS}

BMT recipients, who have undergone total body irradiation used in the conditioning regimen, are at risk of developing radiation pneumonitis. The incidence of symptomatic radiation pneumonitis is approximately $7 \%$; the percentage of patients who manifest asymptomatic radiographic changes is significantly higher. ${ }^{16}$ Dyspnoea is the most common symptom, followed by cough and fever. ${ }^{17}$ Physical findings on chest examination are usually minimal. There is a latent period between radiation exposure and development of pneumonitis, which ranges from one to three months. ${ }^{16}$ In some cases, clinical symptoms may develop even before radiographic changes. ${ }^{18}$ A raised sedimentation rate and leucocytosis are common laboratory findings. Radiographic findings range from mild focal haziness to dense infiltrates, with a relatively sharp border that does not follow the pattern of the lung's anatomical border but rather follow the shape of radiation treatment port. ${ }^{18}$ Computed tomography of the chest shows areas of increased attenuation, with a relatively sharp border like chest radiograph that do not follow the pattern of lung's anatomical border. ${ }^{19}$ Pulmonary function tests reveal restrictive lung physiology and reduced diffusion lung capacity. ${ }^{20}$ Diffusion lung capacity is the most predictive test that may prognosticate the risk of pulmonary radiation damage. ${ }^{20}$ There are many factors that can alter the risk of radiation induced lung damage, these include total radiation dose, fractionation, lung volume, prior radiation, chemotherapy, pre-existing lung disease, and steroid therapy withdrawal. ${ }^{20}$ Mild or subclinical disease, evident by chest radiograph abnormalities, do not require treatment and may need only expectant management. Corticosteroids are the mainstay of treatment for symptomatic patients and usual dose is $1-2 \mathrm{mg} / \mathrm{kg}$ body weight with a gradual taper.

\section{PLEURAL EFFUSION}

Although a large number BMT recipients (40\%-60\%) develop pulmonary complication, there is relatively little information available concerning pleural involvement after BMT. In one study $16 \%$ of patients developed pleural effusions within the first 100 days after receiving $B M T .^{21}$ Perhaps the most common non-infectious cause of pleural effusion is aggressive treatment with fluids, blood, and blood product transfusion. The effusion is usually right sided or bilateral (fig 2 ). In an appropriate setting, thoracentesis is not necessarily required, given the patient has no clinical evidence of infection. Other non-infectious causes of pleural effusion in BMT recipients include parapneumonic effusion, high dose chemotherapy, recurrent malignancy, and iatrogenic. ${ }^{23}$

Hepatic veno-occlusive disease, an occasional complication in patients treated with chemotherapy and radiation therapy for malignant disease, is characterised by jaundice, hepatic enlargement, right upper quadrant pain, and ascites. ${ }^{24} 25$ Pleural effusion has been reported in up to $50 \%$ of BMT recipients with hepatic veno-occlusive disease compared with 3\% of BMT recipients without. ${ }^{26}$ Patients with veno-occlusive disease and pleural effusions have either no or minimal respiratory symptoms. Hepatic veno-occlusive disease usually precedes the development of pleural effusion. The effusion usually resolves without requiring thoracentesis or tube thoracostomy.

\section{MEDIASTINAL EMPHYSEMA}

Mediastinal emphysema can rarely complicate interstitial pneumonitis in BMT recipients and sometimes may precede the transplantation. In a report of six cases, no serious consequences to this problem were noted. ${ }^{27}$ There was a use of higher total dose of irradiation pretransplant in these patients, which might have contributed to the development on the mediastinal emphysema. ${ }^{27}$

\section{VENO-OCCLUSIVE DISEASE}

Veno-occlusive disease is a rare vascular complication of BMT. The clinical picture is dominated by progressive dyspnoea along with signs of pulmonary hypertension and arterial hypoxaemia, six to eight weeks after BMT. ${ }^{28}$ Although clinical 
features and haemodynamic parameters may suggest the diagnosis of veno-occlusive disease, open lung biopsy is required to confirm the diagnosis. The primary pathological changes are occlusive lesions of the small pulmonary venules consisting of fibrous intimal proliferation ${ }^{28}$ High dose chemotherapy and multiple BMTs have been implicated as aetiological factors. Pulmonary veno-occlusive disease may be responsive to high dose steroid therapy when treated promptly. ${ }^{28}$

\section{GRAFT-VERSUS-HOST DISEASE}

GVHD may present as acute or chronic pulmonary process. The incidence of GVHD is significantly higher in allogeneic BMT than in autologous or syngeneic BMT. ${ }^{29}{ }^{30}$ GVHD results from minor incompatibility differences between donor and recipient in HLA matched transplant and is thought to result from an immune reaction mediated by donor T-lymphocytes that recognise the recipient's tissue as a foreign body. ${ }^{5}$ In the early period (first 100 days) after BMT, acute GVHD develops in $25 \%-75 \%$ of patients and primarily affects the skin, liver, and gastrointestinal tract. ${ }^{5}$ Pulmonary complications of acute GVHD are minimal. Chronic GVHD occurs in $20 \%-45 \%$ of BMT recipients who survive six months after transplantation and pulmonary involvement is common in these patients. ${ }^{3031}$ There is a distinct association between the presence of chronic GVHD and increased risk of developing non-infectious pulmonary complications. ${ }^{30} 31$

The predominant respiratory symptoms and signs in chronic pulmonary GVHD patients are dyspnoea, nonproductive cough, crackles, and/or wheezes on auscultation. $^{30}{ }^{31}$ Low grade fever and expectoration are uncommon symptoms..$^{29}$ The median time of onset of respiratory symptoms is five months (range $1-13$ months). ${ }^{30}$ The chest radiograph can be normal despite the presence of clinical symptoms and signs and abnormal pulmonary function tests. $^{32}$ Chest radiography and computed tomography show that pulmonary infiltrates can vary from a few focal areas to diffuse bilateral disease. ${ }^{30}$ Pulmonary function tests demonstrate a fall in vital capacity and forced expiratory volume in the first second, followed by marked decrease in forced expiratory volume in first second/vital capacity ratio suggestive of overt airway obstruction. ${ }^{30}{ }^{31}$ Histologically pulmonary GVHD may manifest as diffuse alveolar damage, lymphocytic interstitial pneumonia, lymphocytic bronchitis, and bronchiolitis obliterans..$^{30} 32$

The majority of patients with GVHD respond to immunosuppressive treatment; this includes high dose steroids (prednisolone $1-2 \mathrm{mg} / \mathrm{kg}$ ) with a gradual taper over 3-12 months in those with sustained complete response. In severe or steroid resistant cases additional immunosuppressive drugs (cyclosporin $\mathrm{A}$, azathioprine, or thalidomide) can be added. ${ }^{29}{ }^{30} \mathrm{In}$ some patients initial favourable response follows a relapsing/ remitting pattern, similar to other autoimmune diseases. ${ }^{3031} 33$

\section{BRONCHIOLITIS OBLITERANS}

Bronchiolitis obliterans is an obstructive pulmonary disorder affecting the small airways that was first described as a late complication of allogeneic BMT, however, it has rarely been described in after autologous BMT. ${ }^{23}$ The incidence of bronchiolitis obliterans has been reported to be between $2 \%$ and $14 \%$ in allogeneic BMT recipients, who survive more than three months. ${ }^{34}$ The BMT recipients with a low posttransplantation immunoglobulin level and those with chronic GVHD are at an increased risk. ${ }^{33}{ }^{34}$

The aetiology of bronchiolitis obliterans remains unclear. Several postulated causes include viral infections, autoimmune processes directed against the bronchial tree, and damage to the small airways secondary to GVHD. ${ }^{34}{ }^{35}$ Although bronchiolitis obliterans is a late onset complication after BMT, it can occur anytime after BMT. ${ }^{34}$ The condition can present subtly, with upper respiratory tract infection symptoms and

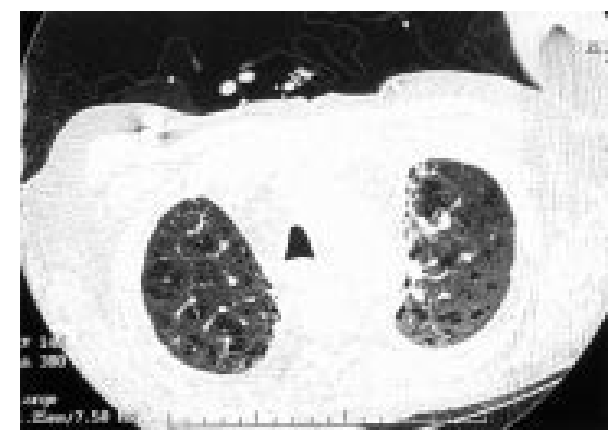

Figure 5 Computed tomogram of the chest in a 51 year old man, four months after autologous BMT, showing areas of attenuation and air trapping suggestive of bronchiolitis obliterans.

gradual dyspnoea accompanied by persistent cough and expiratory wheeze. The chest radiograph shows hyperinflation or is unchanged compared with the baseline. Computed tomography can show a mosaic pattern of attenuation, and evidence of air trapping on an expiratory scan (fig 5 ). ${ }^{36}$

The earliest physiological abnormality is airflow limitation manifested by a decrease in forced expiratory flow at $25 \%$ to $75 \%$ of the forced vital capacity. Diffusing capacity is usually reduced, and occasionally there is a restrictive ventilatory component combined with reduction in exercise capacity. ${ }^{35}$ Progressive airway obstruction with diffusion impairment eventually leads to respiratory failure. Histologically, there is predominately constrictive bronchiolitis with granulation tissue plugs within the small airways, often extending into the alveolar ducts, or complete destruction of the small airways by fibrosis. ${ }^{34}$ The diagnosis of bronchiolitis obliterans can be made based on the appropriate clinical picture, radiographic, and pulmonary function test abnormalities. Histopathological changes are the gold standard for the diagnosis of bronchiolitis obliterans and often require lung biopsy or bronchoscopy with transbronchial biopsy.

The overall prognosis of BMT recipients with bronchiolitis obliterans is dismal, with an overall mortality of $65 \%$ three years after transplantation. ${ }^{33}$ Some authors suggest intensification of the immunosuppressive regimen (high dose prednisone and cyclosporin or azathioprine) to treat this condition; however, the impact of this regimen on the evolution of this disorder is unclear. ${ }^{33}$

SECONDARY PULMONARY ALVEOLAR PROTEINOSIS Secondary pulmonary alveolar proteinosis is another rare complication after BMT, causing potentially reversible respiratory failure. ${ }^{37}$ In a report of 10 patients with immunosuppressive illnesses, including three after BMT, the diagnosis was made on the identification of periodic acid-Schiff positive proteinaceous material from bronchoalveolar lavage with characteristics ultrastructural electron microscopic pattern. ${ }^{37}$ One of the reported patients had reversible disease that coincided with recovery from neutropenia.

\section{SECONDARY MALIGNANCIES}

The incidence of secondary malignancy is seven times that of primary cancer in the general population. ${ }^{38}$ Acute GVHD, total body irradiation, and antithymocyte globulin increase the likelihood of secondary malignancy, while chronic GVHD does not appear to be associated with secondary malignancy. ${ }^{38}$ The chest could be a primary or metastatic site in the case of secondary malignancies.

\section{RADIATION FIBROSIS}

Radiation fibrosis results from radiation induced chronic lung damage and may take six to 24 months to develop. The most 


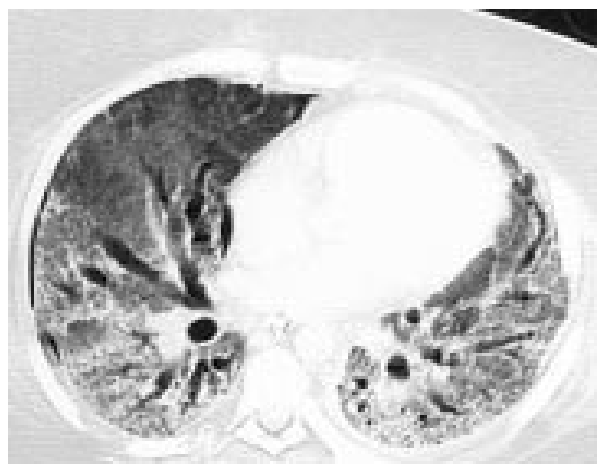

Figure 6 Computed tomography of the chest in a 24 year old woman, seven months after autologous BMT, showing chronic fibrotic changes in both lung fields as well as traction bronchiectasis.

common risk factor for radiation fibrosis is radiation pneumonitis but some patients may develop radiation fibrosis without previous radiation pneumonitis. ${ }^{16}$ Dyspnoea is the most common presenting symptom, and results from pulmonary hypertension and subsequent cor pulmonale. ${ }^{18}$ The severity of symptoms depends on the degree of lung involvement and ranges from asymptomatic to respiratory failure. Chest radiography and computed tomography findings are indistinguishable from pulmonary fibrosis of any other aetiology (fig 6). Serial radiographs can be useful to follow the transition from acute to chronic lung changes. In cases of mild fibrosis, subtle changes - that is, elevation of the hemidiaphragm and minor fissure, apical thickening, and paramediastinal fibrosis with mediastinal widening — can be seen. ${ }^{16}$ In severe cases atelectasis, mediastinal shifting, pleuropericardial adhesions, tenting of the diaphragm, and tracheal deviation can be seen. ${ }^{16}{ }^{18}$ No therapeutic intervention in humans is of proved benefit in radiation induced fibrosis. Supportive measures including supplemental oxygen, bronchodilators, and expectorants can used as needed for symptomatic relief.

\section{PULMONARY CYTOLYTIC THROMBI}

In a recent article, Woodard et al reported a novel noninfectious pulmonary complication in 13 BMT recipients who developed fever and multiple peripheral lung nodules, which were seen on computed tomography. ${ }^{39}$ All patients developed fever at a median of 72 days (range 8-343 days) after BMT, followed by a pulmonary nodule on chest computed tomography. ${ }^{39} 40$ This condition is labelled as "pulmonary cytolytic thrombi". Histological examination of the open lung biopsy showed a unique pattern of necrotic, basophilic thromboemboli amorphous material suggestive of cellular breakdown products occluding medium to large size blood vessels. Immunohistochemical staining showed entrapped leucocytes and disrupted epithelium. No vasculitis, viral inclusions, fungal or bacterial elements were identified. The authors found this condition exclusively in BMT recipients and the majority of the patients were being treated for active GVHD. ${ }^{39}$

\section{PNEUMOTHORAX}

Bronchiolitis obliterans and chronic GVHD after BMT are associated with obstructive airway changes. ${ }^{29-32} 3435$ Spontaneous pneumothorax has been reported in BMT recipients with chronic GVHD and obstructive airway disease. ${ }^{41}{ }^{42}$ Pneumothoraces occurred in patients with advanced to end stage lung disease.

\section{CONCLUSION}

BMT is an effective and promising therapeutic tool for a number of haematological and non-haematological malignant and non-malignant conditions. Its success, however, is often limited by the high incidence of complications, especially those related to the pulmonary system. As the number of BMT recipients increase, the recognition and treatment of pulmonary complications become vital. A better selection of patients, the use of the least toxic conditioning regimens, early recognition and treatment of infectious and non-infectious complications, improved treatment and prevention of GVHD, and more rapid recovery of bone marrow will have a substantial impact on the morbidity and mortality associated with this form of treatment. Although the approach to the diagnosis of pulmonary disease after BMT will often need to be individualised, following a systematic approach is recommended for early diagnosis and appropriate intervention.

\section{Authors' affiliations}

I Khurshid, L C Anderson, Department of Pulmonary and Critical Care Medicine, Brody School of Medicine at East Carolina University,

Greenville, North Carolina, USA

\section{REFERENCES}

1 Krowka MJ, Rosenow EC, Hoagland HC. Pulmonary complications in bone marrow transplantation. Chest 1985;87:237-46.

2 Chan CK, Hyland RH, Hutcheon MA. Pulmonary complications following bone marrow transplantation. Clin Chest Med 1990;11:323-32.

3 Jules-Elysee K, Stover DE, Yahalom J, et al. Pulmonary complications in lymphoma patients treated with high-dose therapy and autologous bone marrow transplantation. Am Rev Respir Dis 1992;146:485-91.

4 Buchner CD, Meyers JD, Springmeyer SC, et al. Pulmonary complications of marrow transplantation. Exp Hematol 1984; 12:1-5.

5 Hamilton PJ, Pearson ADJ. Bone marrow transplantation and the lung Thorax 1986:41:497-502.

6 Dickout WJ, Chan CK, Hyland RH. Prevention of acute pulmonary edema after bone marrow transplantation. Chest 1987;92:303-9.

7 Clark JG, Hansen JA, Hertz MI, et al. Idiopathic pneumonia syndrome after bone marrow transplantation. Am Rev Respir Dis 1993;147:1601-6.

8 Crawford SW, Hackman RC. Clinical course of idiopathic pneumonia after bone marrow transplantation. Am Rev Respir Dis 1993; 147:1393-400

9 Wingard JR, Mellits ED, Sostrin MB, et al. Interstitial pneumonitis after allogeneic bone marrow transplantation. Medicine 1988;67:175-86.

10 Raptis A, Mavroudis D, Suffredini AF, et al. High-dose corticosteroid therapy for diffuse alveolar hemorrhage in allogeneic bone marrow stem cell transplant recipients. Bone Marrow Transplant 1999;24:879-83.

11 Lewis ID, Defor T, Weisdorf DJ. Increasing incidence of diffuse alveolar hemorrhage following allogeneic bone marrow transplantation: cryptic etiology and uncertain therapy. Bone Marrow Transplant 2000;26:539-43.

12 Witte RJ, Gurney JW, Robbins RA, et al. Diffuse pulmonary alveolar hemorrhage after bone marrow transplantation: radiographic findings in 39 patients. AJR Am J Roentgenol 1991;157:461-7.

13 Srivastava A, Gottlieb D, Bradstack KF. Diffuse alveolar hemorrhage associated with microangiopathy after allogeneic bone marrow transplantation. Bone Marrow Transplant 1995;15:863-7.

14 Agustic C, Ramirez J, Picado C, et al. Diffuse alveolar hemorrhage in allogeneic bone marrow transplantation: a postmortem study. Am J Respir Crit Care Med 1995;151:1006-10.

15 Metcalf JP, Rennard SI, Reed EC, et al. Corticosteroids as adjunctive therapy for diffuse alveolar hemorrhage associated with bone marrow transplantation. Am J Med 1994;96:327-34.

16 Movsas B, Raffin TA, Epstein AH, et al. Pulmonary radiation injury. Chest 1997:111:1061-76.

17 Perry MC, Eaton WL, Propert KJ, et al. Chemotherapy with or without radiation therapy in limited small-cell carcinoma of lung. $N$ Engl J Med 1987;316:912-18.

18 Fried JR, Goldberg H. Post-irradiation changes in the lung and thorax. AJR Am J Roentgenol 1940;43:877-95.

19 Panaji JJ, Libshitz HI. CT manifestation of radiation-induced changes in lung tissue. J Comput Assist Tomogr 1982;6:243-8.

20 Catane R, Schwade JG, Turrisi AT III, et al. Pulmonary toxicity after radiation and bleomycin: a review. Int J Radiat Oncol Biol Phys 1979:5:1413-18.

21 Noble PW. The pulmonary complications of bone marrow transplantation in adults (clinical conference). West J Med 1989;150:443-9.

22 Schaap N, Raymaker R, Schatternberg A, et al. Massive pleural effusion attributed to high-dose cyclophosphamide during conditioning for bone marrow transplantation. Bone Marrow Transplant 1996;18:247-47.

23 Schiller G. Chylothorax as a complication central venous catheter-induced superior vena cava thrombosis. Bone Marrow Transplant 1992:9:302.

24 Rollins J. Hepatic venoocclusive disease. Am J Med 1986;81:297-306 
25 Dulley FL, Kanfer EJ, Appelbaum FR, et al. Veno-occlusive disease of the liver after chemotherapy and autologous bone marrow transplantation. Transplantation 1987;43:870-3

26 Ozkaynak MF, Weinberg K, Kohn D, et al. Hepatic veno-occlusive disease post-bone marrow transplantation in children conditioned with busufan and cyclophosphamide: incidence, risk factors and clinical outcome. Bone Marrow Transplant 1991;7:467-76.

27 Hill G, Helenglass G, Powles R, et al. Mediastinal emphysema in marrow transplant recipeints. Bone Marrow Transplant 1987;2:315-20.

28 Hackman RC, Madtes DK, Peterson FB, et al. Pulmonary veno-occlusive disease following bone marrow transplantation. Transplantation 1989;47:989-92.

29 Duncker C, Dohr D, Von Harsdorf S, et al. Non-infectious lung complications are closely associated with chronic graft-versus-host disease: a single center study of incidence, risk factors and outcome. Bone Marrow Transplant 2000;25:1263-8.

30 Schwarer AP, Hughes JMB, Trotman-Dickenson B, et al. A chronic pulmonary syndrome associated with graft-verses-host disease after allogeneic marrow transplantation. Transplantation 1992;6:1002-8.

31 Chan CK, Hyland RH, Hutcheon MA, et al. Small-airway disease in recipients of allogeneic bone marrow transplantation. Medicine 1987;66:327-40.

32 Yousem SA. The histological spectrum of graft-verses-host disease in bone marrow transplant recipients. Hum Pathol 1995;26:668-75.

33 Clark JG, Crawford SW, Madtes DK, et al. Obstructive lung disease after allogeneic marrow transplantation. Ann Intern Med $1989 ; 111: 368-76$
34 Holland $\mathbf{K H}$, Wingard JR, Beschorner WE, et al. Bronchiolitis obliterans in bone marrow transplantation and its relationship to chronic graft-v-host disease and low serum lgG. Blood 1988;72:621-7.

35 Paz HL, Crilley P, Patchefsky A, et al. Bronchiolitis obliterans after autologous bone marrow transplantation. Chest 1992;102:775-8.

36 Worthy SA, Flint JD, Muller NL. Pulmonary complication after bone marrow transplantation: Highresolution $\mathrm{CT}$ and pathologic findings. Radiographics 1997;17:1359-71.

37 Cordonnier C, Fleury-Feith J, Escudier E, et al. Secondary alveolar proteinosis is a reversible cause of respiratory failure in leukemic patients. Am J Respir Crit Care Med 1994;149:788-94.

38 Witherspoon RP, Fisher LD, Schoch G, et al. Secondary cancers after bone marrow transplantation for leukemic or aplastic anemia. N Engl J Med 1989;321:784-9.

39 Woodard JP, Gulbahce E, Shreve M, et al. Pulmonary cytolytic thrombi: a newly recognized complication of stem cell transplantation. Bone Marrow Transplant 2000;25:293-300.

40 Gulbahce HE, Manivel JC, Jessurun J. A previously unrecognized complication of bone marrow transplantation. Mod Pathol $1998 ; 11: 175$ a (abstract).

41 Kurzrock R, Zander A, Kanojia $M$, et al. Obstructive lung disease after allogeneic bone marrow transplantation. Transplantation 1984;37:156-60

42 Link H, Reinhard U, Niethamer D, et al. Obstructive ventilation disorder as a severe complication of chronic graft-versus-host disease after bone marrow transplantation. Exp Hematol 1982;10(suppl 10):92-3.

\section{MEDICAL ANNIVERSARY}

\section{Edward Jenner (17 May 1749)}

- dward Jenner (1749-1823) was born at Berkeley, Gloucestershire, to forebears who were minor gen-

- try and clergymen. He became apprenticed to a doctor at Chipping Sodbury and then became John - Hunter's surgical dresser at St George's Hospital (1770) and his private assistant in Jermyn Street. He practised as a country doctor in Berkeley from 1773 until his discovery of vaccination (1798) and thereafter as a physician at Cheltenham and London. His immortal manuscript is entitled "An inquiry into the Causes and Effects of Variolae Vaccinae, a disease discovered in some of the Western counties of England, particularly Gloucestershire, and known by the name of THE cow Pox" by Edward Jenner.- $D G$ James 